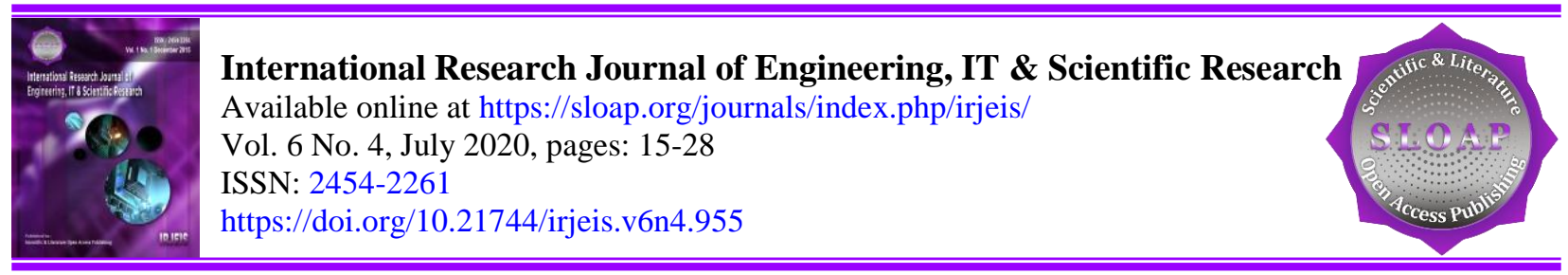

\title{
Mathematical Modeling and Numerical Simulation of Porous Media Single-Phase Fluid Flow Problem: A Scientific Review
}

Article history:

Submitted: 03 January 2020

Revised: 07 March 2020

Accepted: 19 April 2020

\section{Keywords:}

$1 D$ flow; numerical modeling; porous media; reservoir simulation; single-phase flow;

\begin{abstract}
\end{abstract}
The complexity of porous media makes the classical methods used to study hydrocarbon reservoirs inaccurate and insufficient to predict the performance and behavior of the reservoir. Recently, fluid flow simulation and modeling used to decrease the risks in the decision of the evaluation of the reservoir and achieve the best possible economic feasibility. This study deals with a brief review of the fundamental equations required to simulate fluid flow through porous media. In this study, we review the derivative of partial differential equations governing the fluid flow through pores media. The physical interpretation of partial differential equations (especially the pressures diffusive nature) and discretization with finite differences are studied. We restricted theoretic research to slightly compressible fluids, single-phase flow through porous media, and these are sufficient to show various typical aspects of subsurface flow numerical simulation. Moreover, only spatial and time discretization with finite differences will be considered. In this study, a mathematical model is formulated to express single-phase fluid flow in a one-dimensional porous medium. The formulated mathematical model is a partial differential equation of pressure change concerning distance and time. Then this mathematical model converted into a numerical model using the finite differences method. The numerical solution and the mechanism of pressure diffusivity are presented to simulate the studied model.

International research journal of engineering, IT \& scientific research (C) 2020. This is an open access article under the CC BY-NC-ND license (https://creativecommons.org/licenses/by-nc-nd/4.0/).

\section{Corresponding author:}

Yahya Jirjees Tawfeeq,

Petroleum Engineering Department, University of Kirkuk, Kirkuk, Iraq

Email address: yahyapetroleum@uokirkuk.edu.iq

${ }^{a}$ Kirkuk University, Kirkuk, Iraq 


\section{Introduction}

Simulation refers to the science that links the physical concepts and mathematical foundations of a process or phenomenon, supported by computer tool development programming capable of forecasting the behavior and performance of this phenomenon under different practical conditions (Fanchi, 2006; Aarnes et al., 2007). Modeling represents the process of formulating the appropriate model for the simulation process. This mathematical modeling expressed in a way that can be digitally solved to give results that can be analyzed and judge its accuracy by calibrating the model. The simulation of reservoir predicts of reservoir performance under different operating conditions by combining mathematics, physics, reservoir engineering, and computer programming (Jan-Dirk, 2013). Mathematical modeling of a studied phenomenon requires an understanding of the effect of the multiple factors that structure this phenomenon. The simulating of oil reservoirs needed an understanding of the behavior of reservoir rocks (porous medium), and the behavior of reservoir fluids that flow through porous media. Reservoir simulation also required an understanding of the basic concepts of fluid flow such as steady and unsteady flow and the potential energy of fluids (ODEH, 1982). The simulation of reservoirs in the oil industry involves the construction of a petroleum reservoir computer model to improve the estimate of reserves and make decisions for the development of the field. The objectives of simulation studies are to estimate field performance under one or more efficient systems. Model performance observation under various production conditions helps to select an optimum set of production conditions for the reservoir (Hussain \& Alaa, 2017). Numerical simulation of the reservoir is a powerful reservoir engineering tool to expect a reservoir 's future performance under several production conditions. In a single reservoir model, this tool can help engineers to understand the behavior of fluids and rock properties in the reservoir and how these influence the reservoir performance of the future. Depending on the study objectives, several reservoir rocks and fluids can be found from the model (Luca, 2001; Fanchi, 2001; Mustafa et al., 2020). We present in this study the formulation of the mathematical model of isotropic-one-dimension reservoir with single-phase fluid flow. The numerical solution, the application of this model, and the mechanism of pressure diffusivity along with productive formation will be presented to simulate a reservoir with injection and production wells.

\section{Materials and Methods}

\section{Mathematical Models Formulation}

In a porous medium, the single-phase flow mathematical equations describe the physical processes that represent the relationship between the porous medium rocks and the fluids with the applied flow conditions. The general (1, 2, and 3)-dimensional incompressible fluid flow governing equations are; continuity equation, Darcy equation, and equation of state. To formulate a mathematical model for fluid flow through porous media, we start with the definition of continuity equation and control volume concept. The continuity equation is the material balance of mathematical expression. It can be developed in the simple case of one dimensional (1D) flow through considering the mass flowing through a control volume (Aziz, 1979). The control volume (or control box) is a definite volume, fixed in space shape. The shape of the control volume is arbitrary. Its boundaries called control surfaces; they always form a closed surface in space. The control volume form depends on the system of coordinates utilized to define the fluid flow problem. To facilitate discussion, we shall give the control volume a definite shape according to the particular coordinate system employed (e.g., a parallelepiped box in Cartesian coordinates) (BEAR, 1988). Figure (1) shows the finite-control in one-dimensional flow with rectangular coordination. The control volume in petroleum reservoirs simulation applications represents a porous medium comprising single and multi-phases of fluids. The porous media is processed as a continuum, the physical properties of which are represented the medium at any point. The control volume, which depends on the model's coordination system, is selected as shown in Fig. (2) (BEAR, 1988). The equation of material- the balance of any component, c, may be expressed in the system as the law of mass conservation and formulated as (Ertekin et al., 2001; Fanchi, 2006);

$$
\left(m_{i}-m_{o}\right)+\left(m_{s}\right)=m_{a c}
$$

Where; mi = "mass" in, "the mass of the component entering the control volume from other parts of the reservoir"; mo = "mass out" "the mass of the component leaving the control volume to other parts of the reservoir"; ms = "sink/source" "the mass of the component leaving or entering the control volume externally"; and mac = "mass 
accumulated" "the mass of excess material stored in or depleted from the control volume over a time interval $\Delta \mathrm{t}$ ". Also, to express a fluid flow mathematically in a porous medium, a Darcy law and Equation of estate EOS must be used. Darcy's equation is empirical equation describes fluid flow flowing through the control volume.

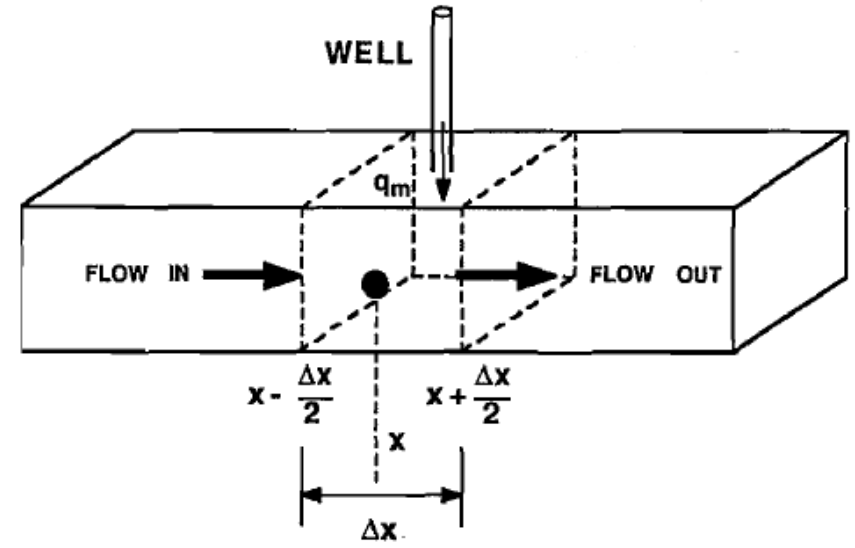

Figure 1. One-dimensional flow control volume in rectangular coordination

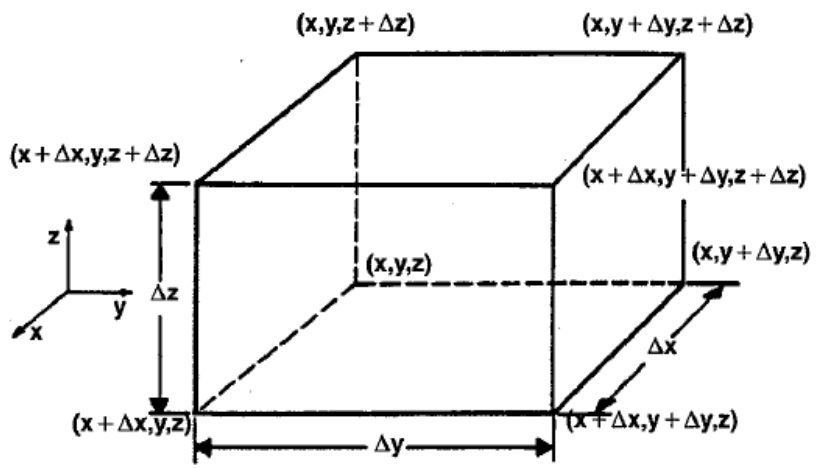

Figure 2. Mathematical symbols description for $1 D$ control volume

For one dimensional (1D) single-phase flow, this equation can be presented in a differential equation as (Peaceman, 2000);

$$
u_{x}=\frac{q}{A_{x}}=\frac{k_{x}}{\mu} \frac{d p}{d x}
$$

Where; ux = "fluid flow rate (q) per unit cross-sectional area (Ax) perpendicular to the flow direction"; $\mathrm{kx}=$ "absolute permeability in the direction of flow"; $\mu=$ "fluid viscosity"; $(\mathrm{dp} / \mathrm{dx})=$ "pressure gradient". Equation of state "EOS" describes the fluid density changes with respects to pressure and temperature and can be expressed as;

$$
\rho_{l}=\rho_{l s}\left[1+C_{l}\left(P-P_{s c}\right)-C_{T l}\left(T-T_{s c}\right)\right]
$$

For a single-phase, we can express the fluid density in term of formation volume factor as (Ertekin et al., 2001);

$$
B_{l}=\frac{\rho_{l s c}}{\rho_{l}}
$$

Where; $C_{l}=$ liquid compressibility; $C_{T l}=$ liquid thermal expansion; $T_{S C}=$ surface temperature; $\mathrm{T}=$ formation temperature; $P_{S C}=$ pressure at surface condition; $\mathrm{P}=$ formation temperature; $B_{l}=$ liquid formation volume factor;

Tawfeeq, Y. J. (2020). Mathematical modeling and numerical simulation of porous media single-phase fluid flow problem: a scientific review. International Research Journal of Engineering, IT \& Scientific Research, 6(4), 15-28. https://doi.org/10.21744/irjeis.v6n4.955 
$\rho_{l s c}=$ fluid density at surface condition; $\rho_{l}=$ fluid density at formation condition. In several studies, many shapes of the control volume assumed when the fluid flows in the porous medium, these shapes vary according to dimensions (1D, 2D and 3D) and according to the type of coordinates (Cartesian, cylindrical, spherical) (BEAR, 1988). In terms of formulating the mathematical model (in its partial differential form) for the single fluid flow, we assumed the control volume in Cartesian coordinates (x, y, z) as shown in Fig. (3) below, to formulate the mathematical model. The mass input or output the control volume amount per unit time is the results of flow rate, q, and density $\rho$ multiplication as;

$$
\left[q\left(\frac{L^{3}}{t}\right)\right] x\left[\rho\left(\frac{m}{L^{3}}\right)\right]=\left[w\left(\frac{m}{t}\right)\right]
$$

Fig. (3) shows a cross-filled arrow representing an extra of fluid volume that entering (or leaving) control volume at mass rate $\mathrm{qm}\left(\frac{L^{3}}{t}\right)$.

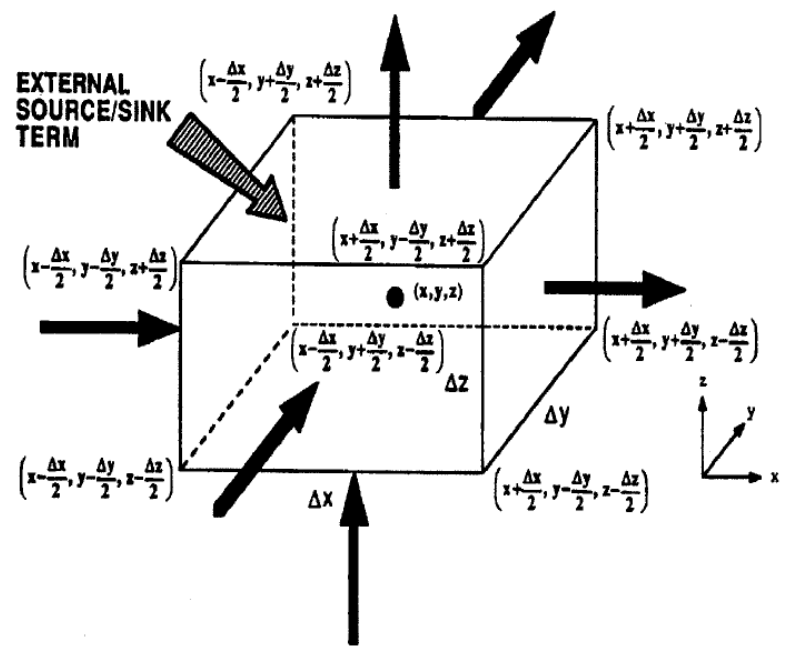

Figure 3. Rectangular coordinates control volume used in this study

The positive sign used for injected fluids and the negative sign used for produced fluid. With these descriptions, the mass-balance equation (Equation 1) can be written for $\mathrm{x}$-direction as;

$$
\left[(W)_{x-\frac{\Delta x}{2}} \Delta t\right]-\left[(W)_{x+\frac{\Delta x}{2}} \Delta t\right]+q_{m} \Delta t=\left[(\varphi \cdot \Delta x \cdot \Delta y \cdot \Delta z \cdot \rho)_{t+\Delta t}-(\varphi \cdot \Delta x \cdot \Delta y \cdot \Delta z \cdot \rho)_{t}\right]
$$

Where; $\varphi=$ formation porosity. The mass flow rate can be defined as;

$$
W_{x}=\dot{m}_{x} \Delta y \Delta z=\dot{m}_{x} A_{x}
$$

Where; $\dot{m}_{x}=$ "the mass flux" (m/L2). The mass flux definition stated in terms of volumetric velocity and density as;

$$
\dot{m}_{x}=\rho u_{x}
$$

Substituting Eq. (7) into Eq. (6) gives;

$$
W_{x}=\rho u_{x} A_{x}
$$

Where; A_x = perpendicular areas to flow lengthways the X-directions. Substituting Eq. (8) into Eq. (5) gives;

$$
-\left[\left(\rho u_{x} A_{x}\right)_{x+\frac{\Delta x}{2}}-\left(\rho u_{x} A_{x}\right)_{x-\frac{\Delta x}{2}}\right]+q_{m}=\frac{1}{\Delta t}\left[(\varphi \cdot \Delta x \cdot \Delta y \cdot \Delta z \cdot \rho)_{t+\Delta t}-(\varphi \cdot \Delta x \cdot \Delta y \cdot \Delta z \cdot \rho)_{t}\right]
$$


Dividing Eq. (9) through by the bulk volume of the control element ( $\mathrm{Vb}=\Delta \mathrm{x} . \Delta \mathrm{y} . \Delta \mathrm{z})$ gives;

$$
-\left[\frac{\left(\rho u_{x} A_{x}\right)_{x+\frac{\Delta x}{2}}-\left(\rho u_{x} A_{x}\right)_{x-\frac{\Delta x}{2}}}{\Delta x}\right]+\frac{q_{m}}{V_{b}}=\frac{(\varphi . \rho)_{t+\Delta t}-(\varphi \cdot \rho)_{t}}{\Delta t}
$$

Take the simultaneous time and space limits;

$$
\lim _{\Delta x ; \Delta t \rightarrow 0}-\left[\frac{\left(\rho u_{x} A_{x}\right)_{x+\frac{\Delta x}{2}}-\left(\rho u_{x} A_{x}\right)_{x-\frac{\Delta x}{2}}}{\Delta x}\right]+\frac{q_{m}}{V_{b}}=\lim _{\Delta x ; \Delta t \rightarrow 0}\left[\frac{(\varphi \cdot \rho)_{t+\Delta t}-(\varphi \cdot \rho)_{t}}{\Delta t}\right]
$$

Eq. (11) represents the spatial first-order partial derivative;

$$
\frac{\partial f}{\partial x}=\lim _{\Delta x \rightarrow 0} \frac{f\left(x+\frac{\Delta x}{2}\right)-f\left(x-\frac{\Delta x}{2}\right)}{\Delta x}
$$

and for time;

$$
\frac{\partial f}{\partial t}=\lim _{\Delta t \rightarrow 0} \frac{f\left(t+\frac{\Delta t}{2}\right)-f(t)}{\Delta t}
$$

Therefore, Eq. (11) can be written as;

$$
-\frac{\partial}{\partial x}\left(\rho u_{x}\right)+\frac{q_{m}}{V_{b}}=\frac{\partial}{\partial t}(\rho \varphi)
$$

Multiplying Eq. (13) by the bulk volume, Vb gives;

$$
-\frac{\partial}{\partial x}\left(\rho u_{x} A_{x}\right) \Delta x+q_{m}=V_{b} \frac{\partial}{\partial t}(\rho \varphi)
$$

The only difference between Eq. (13) and Eq. (14) is that Eq. (13) is written in terms of reservoir bulk volume unit. Eq. (14), on the other hand, is written in terms of the cross-sectional areas perpendicular to the direction of the flow. So, Eq. (14) represents a common equation of the continuity equation in one dimension formulated as a partial derivative Equation. The next step is to integrate the two remaining basic laws, EOS law, and Darcy equation with the continuity equation (Eq. 14). Simply, the EOS law is expressed through using the formation volume factor (FVF) concept, which is represented by Eq. (3b). The formation volume factor is volume under reservoir condition divided by volume under standard conditions (Ahmed, 2010). The velocity terms in Eq. (14) expressed with Darcy's equation, which represents the fluid flow rate through porous media. Before substituting Eq. (2) and Eq. (3b) into Eq. (14), the (qm) term must express as a volumetric rate instead of mass rate.

$$
q_{m}\left(\frac{m}{t}\right)=q_{s c}\left(\frac{L^{3}}{t}\right) \rho_{s c}\left(\frac{m}{L^{3}}\right)
$$

Now substitute Eqs. (2), (3b), and (15) into Eq. (14) to obtain;

$$
\frac{\partial}{\partial x}\left(\frac{A_{x} k_{x}}{\mu B} \frac{\partial p}{\partial x}\right) \Delta x+q_{m}=V_{b} \frac{\partial}{\partial t}\left(\frac{\varphi}{B}\right)
$$

Eq. (16) represents the most general single-phase-flow equation form. We can modify the general flow equation (Eq. 16) according to reservoir fluid type, incompressible, slightly compressible, or compressible fluid (BEAR, 1988). For the incompressible fluid, the density and viscosity are constant, so, Eq. (16) for one-dimensional flow direction can be rewritten as;

Tawfeeq, Y. J. (2020). Mathematical modeling and numerical simulation of porous media single-phase fluid flow problem: a scientific review. International Research Journal of Engineering, IT \& Scientific Research, 


$$
\frac{\partial}{\partial x}\left(A_{x} k_{x} \frac{\partial p}{\partial x}\right) \Delta x+\mu q_{s c}=0
$$

For slightly compressible liquid flow, the FVF can be expressed as;

$$
B=\frac{B^{o}}{\left[1+C_{l}\left(p-p^{0}\right)\right.}
$$

Where $\mathrm{c}=$ fluid compressibility. Substituting of Eq. (18) in Eq. (16), and assuming incompressible porous medium, yields;

$$
V_{b} \frac{\partial}{\partial t}\left(\frac{\varphi}{B}\right)=V_{b} \varphi \frac{\partial}{\partial t}\left(\left[\frac{1+C_{l}\left(p-p^{o}\right)}{B^{o}}\right]\right)=\frac{V_{b} \varphi C_{l}}{B^{o}} \frac{\partial p}{\partial t}
$$

The one-dimensional slightly compressible fluid flow equation then becomes;

$$
\frac{\partial}{\partial x}\left(\frac{A_{x}}{\mu} \frac{k_{x}}{B} \frac{\partial p}{\partial x}\right) \Delta x+q_{s c}=\frac{V_{b} \varphi C_{l}}{B^{o}} \frac{\partial p}{\partial t}
$$

Substituting Eq. (18) on the left side of Eq. (20) and assuming $\mu$ as a constant, Eq. (20) then becomes;

$$
\frac{\partial}{\partial x}\left(A_{x} k_{x}\left[1+C_{l}\left(p-p^{0}\right) \frac{\partial p}{\partial x}\right) \Delta x+B^{o} \mu q_{s c}=V_{b} \varphi \mu C_{l} \frac{\partial p}{\partial t}\right.
$$

In many cases, for slightly compressible fluids we can assume $\left[1+\mathrm{C}_{-} 1\left(\mathrm{p}-\mathrm{p}^{\wedge} 0\right) \approx 1\right.$ because $(\mathrm{Cl})$ is very small. So;

$$
\frac{\partial}{\partial x}\left(A_{x} k_{x} \frac{\partial p}{\partial x}\right) \Delta x+B^{o} \mu q_{s c}=V_{b} \varphi \mu C_{l} \frac{\partial p}{\partial t}
$$

Eq. (22) represents the one-dimensional slightly compressible fluid flow for single-phase, in "heterogeneous" and "anisotropic" formation. For "homogeneous" and "isotropic" formation, the Equation rearranged to get;

$$
\frac{\partial^{2} p}{\partial x^{2}}+\frac{B^{o} \mu}{V_{b} k_{x}} q_{s c}=\frac{\varphi \mu C_{l}}{k_{x}} \frac{\partial p}{\partial t}
$$

Also, in absence of external source (qin=qout=0), Eq. (23) becomes;

$$
\frac{\partial^{2} p}{\partial x^{2}}=\frac{\varphi \mu C_{l}}{k_{x}} \frac{\partial p}{\partial t}
$$

Equation (24) is slightly compressible- the flow equation represents a time-dependent problem.

\section{Numerical Approximation}

In the previous section, equations (Eq. 20) are derived for single-phase slightly compressible fluid flow through the porous media. This equation was spatial second-order partial differential equations and time first-order partial differential equations. In general, the analytically (exactly) solution for this equation is very hard and complex because of equation nonlinearity nature (Mattax \& Dalton, 1990). To solve this type of equation, a numerical approximate method is needed (Aarnes, 2007). The finite-difference method was a common numerical technique currently in use in the oil industry (ODEH, 1982; Gerritsen \& Durlofsky, 2005). However, the governing equations (Eq. 20) used to describe flow in porous media only contain first-order derivatives, and we, therefore, limit our study to first-order derivatives. We use the truncated Taylor series expansion to approximate first-order derivatives. The hypothetical pressure distribution (P) is shown in Fig. (4) along x-direction. The derivative approximated at point $\mathrm{x}$ (referred also as i) on which the pressure value is referred as (Pi). There are two adjacent points to the central point 
$(\mathrm{Xi}-1)$ and $(\mathrm{Xi}-1)$. Therefore, the pressure values at these two adjacent nodes are $(\mathrm{Pi}+1)$ and $(\mathrm{Pi}-1)$ respectively. The first-order derivative forward-difference approximation uses values of the function at points (i) and $(i+1)$ to get;

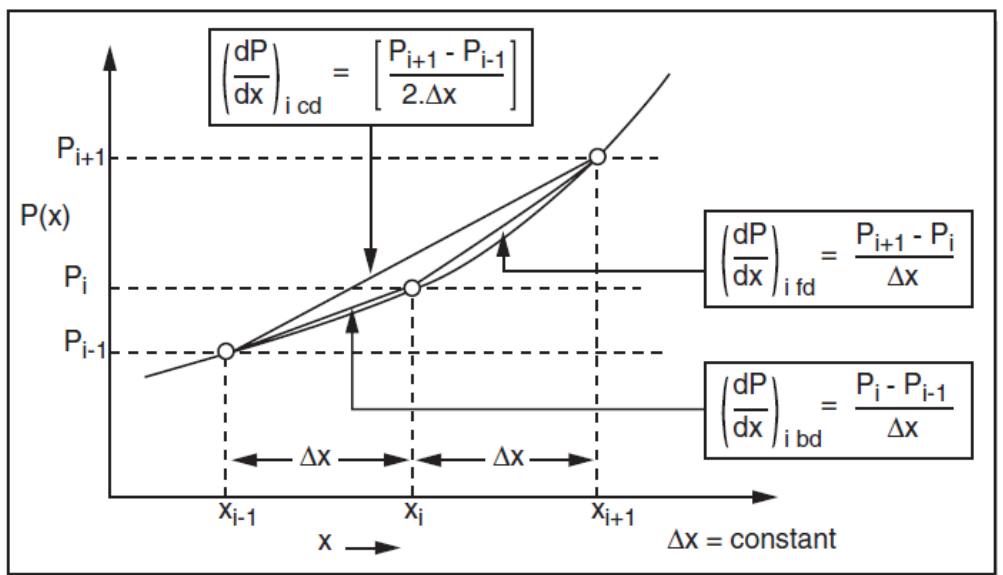

Figure 4. Graphical illustration of the finite difference derivatives calculated by backward differences, forward differences and central differences

$$
\left(\frac{\partial p}{\partial x}\right)_{i}=\frac{p_{i+1}-p_{i}}{\Delta x}
$$

If this derivative is written at point (i) in the spatial domain, it indicating the pressure change rate with the time at point (i). or;

$$
\left(\frac{\partial p}{\partial x}\right)_{i}=\frac{p_{i}^{n+1}-p_{i}^{n}}{\Delta t}
$$

We can achieve a backward difference approach to the first-order derivative using the same way, but now using a neighboring point;

$$
\left(\frac{\partial p}{\partial x}\right)_{i}=\frac{p_{i}-p_{i-1}}{\Delta x}
$$

The central difference approximation to the first-order derivative at point (i) uses the two adjacent points as;

$$
\left(\frac{\partial p}{\partial x}\right)_{i}=\frac{p_{i+1}-p_{i-1}}{2 \Delta x}
$$

The algebraic finite-difference equations result from the implementation of finite-difference approximations (Ewing, 1983). The solutions for finite differences equations can only be achieved at discrete grid-system points. This means the calculated pressure from the simulator of the reservoir is only known in grid points within the reservoir. Unlike the solutions of the continuous equation obtained at all points in the reservoir. Generally, discretization is a process by which the continuous equations are converted into the finite-difference equations (Ertekin et al., 2001). Two forms of finite-difference grids utilized in the simulation of the reservoir: block centered and point distributed (Chen et al., 2006). In a rectangular block-centered grid, the grid points defined as the centers of these grid blocks (as shown in Fig. 5b). In a rectangular point-distributed grid, a block boundary placed at the corner point of the grid block (as shown in Fig. 5a). The grid system aims to divide the reservoir into blocks that representational rock properties allocated. Therefore, the grid cells should be small sufficient to define reservoir heterogeneity and to adequately represent the flow behavior of the average grid cell properties in the reservoir (Ertekin et al., 2001). Using the block-centered grid, we can discretize Eq. (20) by the central difference approximation as;

Tawfeeq, Y. J. (2020). Mathematical modeling and numerical simulation of porous media single-phase fluid flow problem: a scientific review. International Research Journal of Engineering, IT \& Scientific Research, 6(4), 15-28. https://doi.org/10.21744/irjeis.v6n4.955 


$$
\frac{\partial}{\partial x}\left(\frac{A_{x}}{\mu_{l}} \frac{k_{x}}{B_{l}} \frac{\partial p}{\partial x}\right)_{i} \cong \frac{1}{\Delta x_{i}}\left[\left(\frac{A_{x}}{\mu_{l}} \frac{k_{x}}{B_{l}} \frac{\partial p}{\partial x}\right)_{i+\frac{1}{2}}-\left(\frac{A_{x}}{\mu_{l}} \frac{k_{x}}{B_{l}} \frac{\partial p}{\partial x}\right)_{i-\frac{1}{2}}\right]
$$

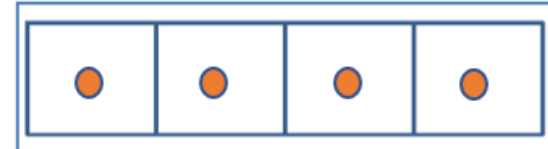

(b)

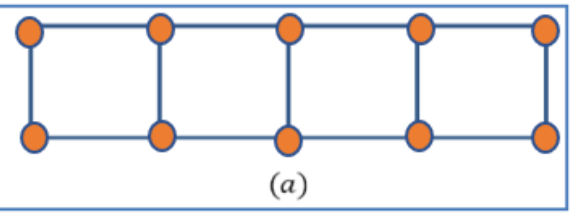

(a)

Figure 5. One-dimensional grid system: (a) point distributed grid system, (b) block centered grid system

Eq. (29) is used to approximate the spatial derivative at grid point (i) and the result is substituted into the left side of Eq. (20) to yields;

$$
\frac{1}{\Delta x_{i}}\left[\left(\frac{A_{x}}{\mu_{l}} \frac{k_{x}}{B_{l}} \frac{\partial p}{\partial x}\right)_{i+\frac{1}{2}}-\left(\frac{A_{x}}{\mu_{l}} \frac{k_{x}}{B_{l}} \frac{\partial p}{\partial x}\right)_{i-\frac{1}{2}}\right] \Delta x_{i}+q_{l s c}=\left(\frac{V_{b} \varphi C_{l}}{B_{l}^{o}} \frac{\partial p}{\partial t}\right)_{i}
$$

Now, using of central differences to approximate $\left(\frac{\partial p}{\partial x}\right)_{i+\frac{1}{2}}$ and $\left(\frac{\partial p}{\partial x}\right)_{i-\frac{1}{2}}$ yields;

$$
\begin{aligned}
& \left(\frac{\partial p}{\partial x}\right)_{i+\frac{1}{2}}=\frac{p_{i+1}-p_{i}}{x_{i+1}-x_{i}}=\frac{p_{i+1}-p_{i}}{\Delta x_{i+\frac{1}{2}}} \\
& \left(\frac{\partial p}{\partial x}\right)_{i-\frac{1}{2}}=\frac{p_{i}-p_{i-1}}{x_{i}-x_{i-1}}=\frac{p_{i}-p_{i-1}}{\Delta x_{i-\frac{1}{2}}}
\end{aligned}
$$

Substituting Eqs. (31) and (32) into Eq. (30) results in;

$\left(\frac{A_{x}}{\mu_{l}} \frac{k_{x}}{B_{l} \Delta x}\right)_{i+\frac{1}{2}}\left(p_{i+1}-p_{i}\right)-\left(\frac{A_{x}}{\mu_{l}} \frac{k_{x}}{B_{l} \Delta x}\right)_{i-\frac{1}{2}}\left(p_{i}-p_{i-1}\right)+q_{l s c}=\left(\frac{V_{b} \varphi C_{l}}{B_{l}^{o}}\right)_{i} \frac{\partial p_{i}}{\partial t}$

Or;

$T_{l_{i+\frac{1}{2}}}\left(p_{i+1}-p_{i}\right)-T_{l_{i-\frac{1}{2}}}\left(p_{i}-p_{i-1}\right)+q_{l s c}=\left(\frac{V_{b} \varphi C_{l}}{B_{l}^{O}}\right)_{i} \frac{\partial p_{i}}{\partial t}$

The coefficients $T_{l_{i+\frac{1}{2}}}$ and $T_{l_{i-\frac{1}{2}}}$ are referred to as the transmissibility of the porous medium, and is considered to be porous medium property. For a uniform block-centered grid, spacings between the grid points are identical and equal to the block dimension $\Delta \mathrm{x}$. So;

$$
\Delta x_{i+\frac{1}{2}}=\Delta x_{i-\frac{1}{2}}=\Delta x_{i}=\Delta x
$$

Eq. (33) and Eq. (34) indicate that, the pressures $(\mathrm{Pi}+1)$ and $(\mathrm{Pi}-1)$ must be known to solve for pressure (pi). So, Eq. (34) must be written to each grid block in reservoir simulation modeling. This yields system of equations that approximates Eq. (20). The discretization of the time derivative $\left(\frac{\partial p_{i}}{\partial t}\right)$ in Eqs. (33) and (34) is treated in the same way as the spatial derivative $\left(\frac{\partial p}{\partial x}\right)_{i}$. The backward-difference approximation commonly is utilized in reservoir simulation for the discretization of the time derivative $\frac{\partial p_{i}}{\partial t}$ ). The backward-difference approximation to the first derivative at base time level $\mathrm{tn}+1$ defined as;

$$
\frac{\partial p}{\partial t} \approx \frac{p\left(t^{n+1}\right)-p\left(t^{n}\right)}{\Delta t}
$$


With the notation;

$p^{n}=p\left(t^{n}\right)$

$p^{n+1}=p\left(t^{n+1}\right)$

Eq. (36) written for grid point (i) with the sign of equal rather than a sign of approximation becomes;

$\frac{\partial p_{i}}{\partial t}=\frac{p_{i}^{n+1}-p_{i}^{n}}{\Delta t}$

Substituting into Eq. (33) at time level tn+1 results in;

$\left(\frac{A_{x}}{\mu_{l}} \frac{k_{x}}{B_{l} \Delta x}\right)_{i+\frac{1}{2}}\left(p_{i+1}^{n+1}-p_{i}^{n+1}\right)-\left(\frac{A_{x}}{\mu_{l}} \frac{k_{x}}{B_{l} \Delta x}\right)_{i-\frac{1}{2}}\left(p_{i}^{n+1}-p_{i-1}^{n+1}\right)+q_{l s c_{i}}=\left(\frac{V_{b} \varphi C_{l}}{B_{l}^{o} \Delta t}\right)_{i}\left(p_{i}^{n+1}-p_{i}^{n}\right)$

or, in terms of the transmissibility yields;

$T_{i+\frac{1}{2}}\left(p_{i+1}^{n+1}-p_{i}^{n+1}\right)-T_{i-\frac{1}{2}}\left(p_{i}^{n+1}-p_{i-1}^{n+1}\right)+q_{l s c_{i}}=\left(\frac{V_{b} \varphi C_{l}}{B_{l}^{o} \Delta t}\right)_{i}\left(p_{i}^{n+1}-p_{i}^{n}\right)$

Note that from Eqs. (39) and (40), all pressures on the left side of the Eq. (40) given in the time level tn+1 are the unknowns. If we evaluate the transmissibility at $(\mathrm{pn}+1)$, the coefficients of the equation are functions of unknowns and resulting in a nonlinear algebraic equation that needs further modification before solving it with an appropriate linear-equation solver. Such nonlinear problems arise in the slightly compressible single-phase fluid flow. On the other hand, the transmissibility evaluation at (pn) with assumption the base time level to be (tn), the coefficients of the equation can be evaluated explicitly with the known pressures. We will currently use the explicit treatment with the forward difference approximation to write Eq. (39) at time level (tn) as;

$\left(\frac{A_{x}}{\mu_{l}} \frac{k_{x}}{B_{l} \Delta x}\right)_{i+\frac{1}{2}}^{n}\left(p_{i+1}^{n}-p_{i}^{n}\right)-\left(\frac{A_{x}}{\mu_{l}} \frac{k_{x}}{B_{l} \Delta x}\right)_{i-\frac{1}{2}}^{n}\left(p_{i}^{n}-p_{i-1}^{n}\right)+q_{l s c_{i}}=\left(\frac{V_{b} \varphi C_{l}}{B_{l}^{o} \Delta t}\right)_{i}\left(p_{i}^{n+1}-p_{i}^{n}\right)$

or, in terms of the transmissibility;

$T_{i+\frac{1}{2}}^{n}\left(p_{i+1}^{n}-p_{i}^{n}\right)-T_{i-\frac{1}{2}}^{n}\left(p_{i}^{n}-p_{i-1}^{n}\right)+q_{l s c_{i}}=\left(\frac{V_{b} \varphi C_{l}}{B_{l}^{o} \Delta t}\right)_{i}\left(p_{i}^{n+1}-p_{i}^{n}\right)$

Note that from Eqs. (41) and (42), all pressures to the flow equation are known except Pn+1 at the time level (tn). The approximation of forwarding difference of flow equation outcomes in an explicit evaluation procedure for unknown pressure $(P n+1)$ can be expressed as;

$p_{i}^{n+1}=p_{i}^{n}+\left(\frac{B_{l}^{o} \Delta t}{V_{b} \varphi C_{l}}\right)_{i} q_{l s c_{i}}+\left(\frac{B_{l}^{o} \Delta t}{V_{b} \varphi C_{l}}\right)_{i}\left[T_{i+\frac{1}{2}}^{n} p_{i+1}^{n}-\left(T_{i+\frac{1}{2}}^{n}+T_{i-\frac{1}{2}}^{n}\right) p_{i}^{n}+T_{i-\frac{1}{2}}^{n} p_{i-1}^{n}\right]$

With;

$T_{i \pm \frac{1}{2}}=\left(\frac{A_{x}}{\mu_{l}} \frac{k_{x}}{B_{l} \Delta x}\right)_{i \pm \frac{1}{2}}$

Note that from Eq. (43), all term on the right side of Eq. (43) is known since all pressure appearing on this site are at the known old-time level $\left(\mathrm{P}^{\mathrm{n}}\right)$.

\section{Initial / Boundary Conditions}

The conditions of initial and boundary represent the supplementary data needed to get a unique solution to a given differential equation. The initial condition required for reservoir simulation problems is initial reservoir pressure. The boundary conditions requirements include exterior boundaries (limits of the reservoir) and interior boundaries (production and injection wells). The "no-flow boundary" occurs at some distance from the reservoir for external boundaries is generally assumed. This can be stated mathematically as;

Tawfeeq, Y. J. (2020). Mathematical modeling and numerical simulation of porous media single-phase fluid flow problem: a scientific review. International Research Journal of Engineering, IT \& Scientific Research,

6(4), 15-28. https://doi.org/10.21744/irjeis.v6n4.955 


$$
\frac{\partial p}{\partial x}=0
$$

When all gradients $(\mathrm{dp} / \mathrm{dx})$ across reservoir boundaries are equal to zero, the reservoir boundary is assumed to be "no flow-free border". Boundary conditions that defined a derivative on a boundary denoted as "Neumann-type boundary" conditions (Ertekin et al., 2001).

\section{Results and Discussions}

This paper presents a numerical method and mathematical study for one dimensional (1D) single-phase fluid flow through porous media. We have solved the mathematical models (Eq. 20) describing the single-phase flow problem, in cartesian coordinates which are solved using a finite difference method as described in previous sections. In this study, as application examples, we consider the one-dimensional (1D) block-centered grid with no boundary flow, shown in Fig. (6). As shown in Fig. (6), the geometrical network of the studied reservoir is discretized into four blocks with a rectangular block-centered grid system. The injection well is located at the grid block number (1), while the production well is located at grid block number (4). The exterior boundaries condition assumed that noflow boundary exists, i.e. all gradients (dp/dx) across reservoir boundary are equal to zero. Reservoir properties and grid block dimensions are given in table (1).

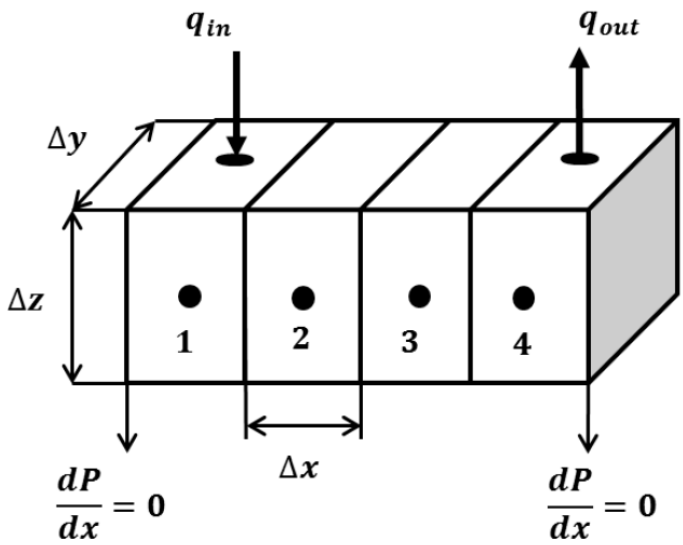

Figure 6. Porous medium and block-centered grid system used in this study

Table 1

Summary of reservoir and grid parameters calculated from block properties $x$-direction block dimension $\quad \Delta \mathrm{x} \quad 800 \mathrm{ft}$ $y$-direction block dimension $\quad \Delta \mathrm{y} \quad 800 \mathrm{ft}$ $z$-direction block dimension $\quad \Delta \mathrm{z} \quad 100 \mathrm{ft}$ Porosity

Permeability in direction of $x$-axis Compressibility for Phase $l$

The viscosity of Phase $l$ time step Injection rate

Production rate

Initial reservoir pressure

Reservoir bulk volume $(\Delta \mathrm{x} . \Delta \mathrm{y} . \Delta \mathrm{z})$

Cross-sectional area of $x$-direction $(\Delta \mathrm{y} . \Delta \mathrm{z})$

$\varphi \quad 0.2$

$\mathrm{Kx} \quad 50 \mathrm{md}$

$\mathrm{C}_{l} \quad 5 \times 10^{-6 \mathrm{psi}-1}$

$\mu_{l} \quad 15 \mathrm{cp}$

$\Delta \mathrm{t} \quad 15$ day

q $\quad 150(\mathrm{STB} /$ day $)$

qout 200 (STB/day)

$\mathrm{P}_{\text {in }} \quad 8000 \mathrm{psi}$

$\mathrm{V}_{\mathrm{b}} \quad 64 \times 10^{6} \mathrm{ft}^{3}$

$\mathrm{A}_{\mathrm{x}} \quad 80 \times 10^{3} \mathrm{ft}^{2}$

The computed code for numerical approximation (Eqs. 41 through 44) is written in Microsoft Excel 2019 Visual Basic Applications (VBA) as shown in Fig. (7). 


\begin{tabular}{|c|c|c|c|c|c|c|c|c|c|c|}
\hline Project - VBAProject & $x$ & CommandButton1 & & & & & & & & \\
\hline 國圆回 & & Private Sub ConnandButton1_Click|) & G21 & 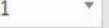 & $x$ & $f_{x}$ & & & & \\
\hline 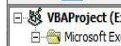 & $\begin{array}{l}\text { Explicit No Flow.xls) } \\
\text { Excel Otjects }\end{array}$ & $\begin{array}{l}\text { Dim PI (100), E }(100), \mathrm{I}(100 \overline{\bar{l}}, \mathrm{Q}(30) \text { A.s Single } \\
\text { Rem Nb is the No. of Block }\end{array}$ & $\Delta$ & $\mathrm{B}$ & C & D & $E$ & $\mathrm{~F}$ & G & $\mathrm{H}$ \\
\hline 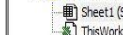 & $\begin{array}{l}1 \text { (sheet1) } \\
\text { akbook }\end{array}$ & $\begin{array}{l}\text { No }=\operatorname{Cel1s}(5,2) \\
\text { Nt }=\operatorname{Int}(\operatorname{Cel1s}(6,2) / \operatorname{Cel1s}(7,2))\end{array}$ & 2 & & & & & & & \\
\hline & & $\begin{array}{l}d t=\text { Cel1s }(7,2) \\
\text { Rem INOUT DATR }\end{array}$ & 3 & EXPLICT & & Clear Be & or Run & & Run & \\
\hline & & 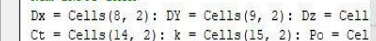 & 4 & & & & & & & \\
\hline & & 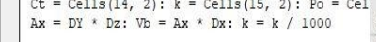 & 5 & 4 & & $\mathrm{x}$ & 800 & 1600 & 2400 & 3200 \\
\hline & & Rem C1 $=\operatorname{ALF} \times \mathrm{B} 1 \times \mathrm{DL} /(\mathrm{Wb} \times \mathrm{PD} \times \mathrm{Ct})$ & 6 & 180 & & Time,Days & Block 1 & Block 2 & Block 3 & Block 4 \\
\hline Properties - Shet1 & $x$ & $\begin{array}{l}\mathrm{C}=\mathrm{ALF} * \mathrm{Bl} * \mathrm{dt} /(\mathrm{Vo} * \mathrm{Po} * \mathrm{Ct}) \\
\text { Rem Constant of Transmissibility } \mathrm{C2}\end{array}$ & 7 & 15 & Initial P & 0 & 8000 & 8000 & 8000 & 8000 \\
\hline Sheet1 Woksheet &. & $C 2=B C * A x \times k /(O * D x)$ & 8 & 800 & & 15 & 8035.16 & 8000.00 & 8000.00 & 7953.13 \\
\hline 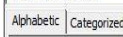 & & For $I=1$ To $\mathbb{N b}$ & 9 & 800 & & 30 & 8067.57 & 8002.75 & 7996.34 & 7909.91 \\
\hline \begin{tabular}{|l|} 
(Name) \\
Cisplyy PagBbereks
\end{tabular} & $\begin{array}{l}\text { Sheet1 } \\
\text { False }\end{array}$ & $\begin{array}{l}T(1)=c 2 \\
\text { Nexe I }\end{array}$ & 10 & 100 & & 45 & 8097.66 & 8007.31 & 7990.09 & 7869.79 \\
\hline DisplyyPagebreaks & $\begin{array}{l}\text { False } \\
\text { False } \\
\text { Ealoe }\end{array}$ & $T(0)=0: T(N b+1)=0$ & 11 & 1 & & 60 & 8125.76 & 8013.02 & 7982.03 & 7832.31 \\
\hline
\end{tabular}

Figure 7. Numerical approximation code was written by Excel VBA interface

This code was programmed to fit the discretized studied reservoir to obtain accurate pressure distributions results from numerical approximation for six months from the starting of injection and production operations. Reservoir properties and grid blocks dimensions input parameters are classified into six groups of input data, as shown in Fig. (8);

1) Grid blocks dimensions (reservoir dimensions) to calculate cross-sectional drainage area in (x) direction (Ax) and reservoir bulk volume ( $\mathrm{Vb}$ ).

2) Reservoir petrophysical properties such as porosity and permeability data.

3) Flowing fluid properties, such as viscosity and compressibility data.

4) Reservoir initial pressure data.

5) Injection and production well flow rate data.

6) Time steps data.

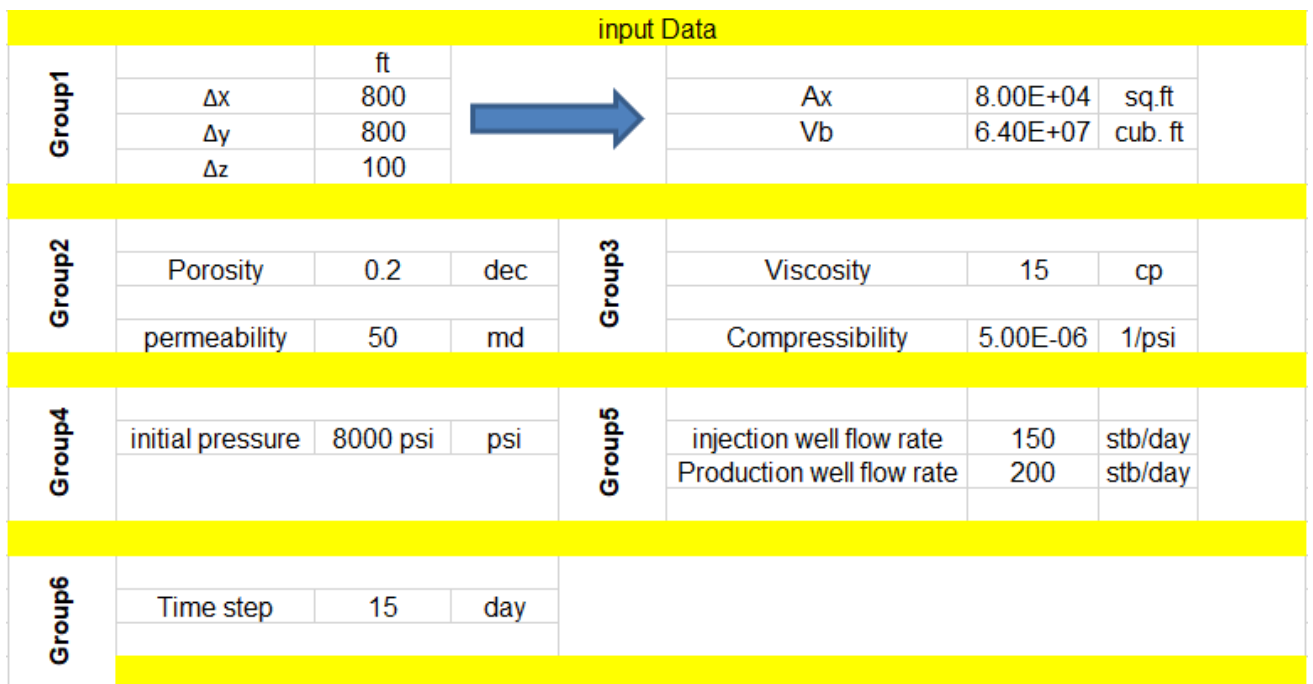

Figure 8. Reservoir and grid blocks input parameter classification

The Explicit simulation-study final results for pressure distribution during six months are listed in the table (2). Fig. (9) and Fig. (10) shows the pressure distributions for four grid blocks between injection and production wells during 15, 30, 45, 90, and 150 days. From these figures, we note that the pressure before starting the injection and production $(\mathrm{t}=0$ days) is constant and equal to the initial reservoir pressure $(\mathrm{p}=8000 \mathrm{psi})$ for all blocks. At the time step $(t=15$ days $)$, i.e., after fifteen days from the start of production and injection operations, the pressure increased to $(p=8035 \mathrm{psi})$ in the block (1) where the injection well is located. Fluid injection into the reservoir increases the reservoir energy, and therefore the reservoir pressure will increase. In block (2) and block (3), the pressure value remains constant and equal to the initial reservoir pressure value ( $p=8000 \mathrm{psi}$ ), as these blocks were not affected either by the injection rate in the block (1) or the production rate in the block (4). Whereas in the block (4) where the

Tawfeeq, Y. J. (2020). Mathematical modeling and numerical simulation of porous media single-phase fluid flow problem: a scientific review. International Research Journal of Engineering, IT \& Scientific Research,

6(4), 15-28. https://doi.org/10.21744/irjeis.v6n4.955 
production well is located, we notice a decrease in pressure to the value $(\mathrm{p}=7953 \mathrm{psi}$ ) because fluid production from the reservoir decreases the reservoir energy and therefore the reservoir pressure will drop. After a month $(\mathrm{t}=30$ days) of production and injection operations starting, we notice that the pressure rises to $(\mathrm{p}=8067 \mathrm{psi})$ in the block (1) where the injection well is located, and in the block (2) the pressure increases to ( $p=8002.7$ psi) as a result of being affected by the pressure of injection well in the block (1). In block (3), we note the decrease in pressure to (7996.3) because this block is adjacent to the producing well and it was affected by the pressure drop in the block (4). The pressure change (increase and decrease) continues over time with injection and production operations. Thus, through the used grid system model, we can determine the volume of injected fluid needed to obtain the pressure change at the desired limits to get the required production rate.

Table 2

Explicit simulation-study final results for pressure distribution for six months

\begin{tabular}{cccccc}
\hline & $\begin{array}{c}\text { X, ft } \\
\text { Time, Days }\end{array}$ & $\begin{array}{c}800 \\
\text { Block 1 }\end{array}$ & $\begin{array}{c}1600 \\
\text { Block 2 }\end{array}$ & $\begin{array}{c}2400 \\
\text { Block 3 }\end{array}$ & $\begin{array}{c}3200 \\
\text { Block 4 }\end{array}$ \\
\hline Initial P & 0 & 8000 & 8000 & 8000 & 8000 \\
& 15 & 8035.16 & 8000.00 & 8000.00 & 7953.13 \\
30 & 8067.57 & 8002.75 & 7996.34 & 7909.91 \\
& 45 & 8097.66 & 8007.31 & 7990.09 & 7869.79 \\
60 & 8125.76 & 8013.02 & 7982.03 & 7832.31 \\
75 & 8152.10 & 8019.41 & 7972.76 & 7797.13 \\
90 & 8176.89 & 8026.13 & 7962.68 & 7763.98 \\
105 & 8200.27 & 8032.95 & 7952.12 & 7732.63 \\
120 & 8222.36 & 8039.71 & 7941.28 & 7702.90 \\
135 & 8243.24 & 8046.29 & 7930.35 & 7674.65 \\
150 & 8263.01 & 8052.62 & 7919.43 & 7647.75 \\
165 & 8281.73 & 8058.65 & 7908.61 & 7622.10 \\
180 & 8299.46 & 8064.36 & 7897.95 & 7597.61 \\
\hline
\end{tabular}

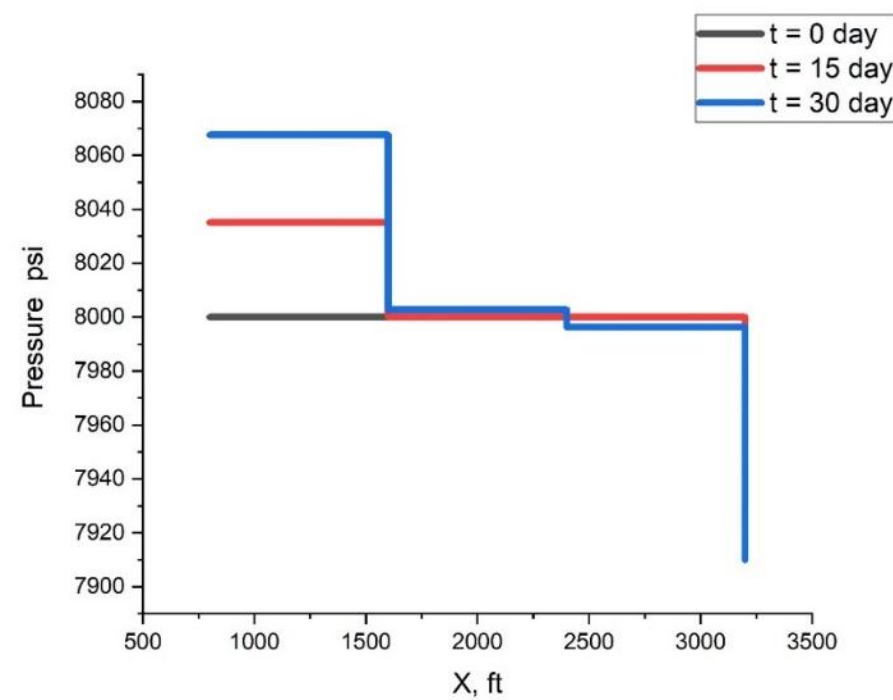

Fig. 9. Pressure distributions in the reservoir between injection and production wells for time steps 15 and 30 days for all blocks 


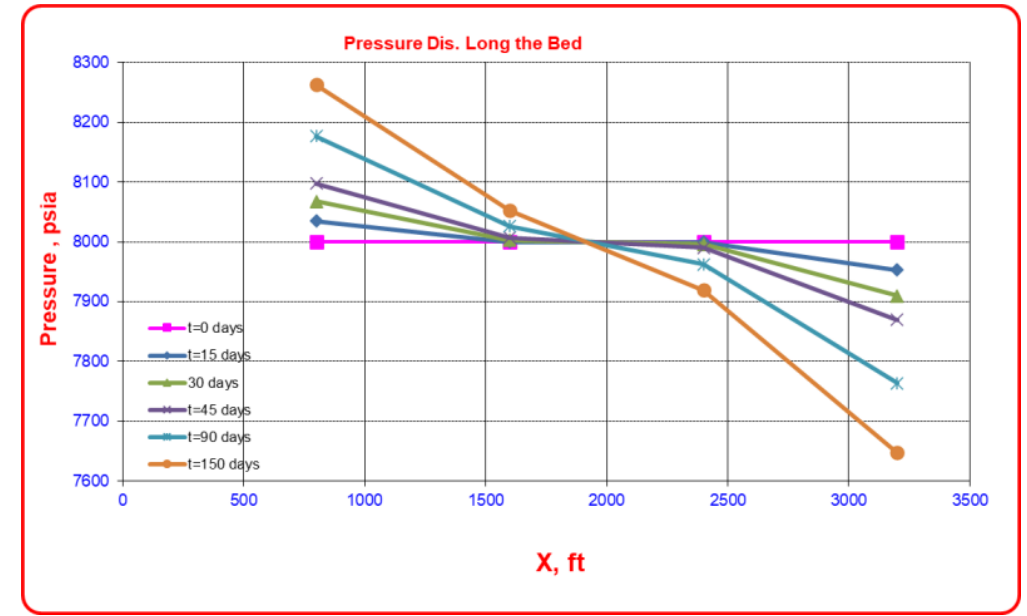

Figure 10. Pressure distributions in the reservoir between injection and production wells for time step 15, 30, 45, 90 and 150 days for all blocks

\section{Conclusion}

The main conclusions of the current study can be summarized as follows:

1) The physical concepts of fluid flow through the porous medium such as; Darcy law, conservation of mass law, and Equation of state were combined to obtain the mathematical model of single-phase fluid flow in a one-dimensional porous medium.

2) The obtained mathematical model (Eq. 20), is a partial differential equation (second order in space and firstorder in time).

3) The conversion of the mathematical model into a numerical model was done using the finite difference method, which enabled us to find an approximate solution for the derivatives.

4) The pressure distributions through the reservoir blocks are essential to determine the production indicators and select the locations of injection and production wells.

5) The adjacent blocks to injection and production wells are not affected by pressure changes during the early periods of injection and production operations.

6) Pressure changes begin to affect the adjacent blocks to the injection and production wells after a specific time (after one month in our studied model).

7) Fluid injection into the reservoir increases the reservoir energy, and therefore the reservoir pressure will increase.

Fluid production from the reservoir decreases the reservoir energy, and therefore the reservoir pressure will drop.

Conflict of interest statement

The author declared that he has no competing interests.

Statement of authorship

The author has a responsibility for the conception and design of the study. The author has approved the final article.

Acknowledgments

I am grateful to two anonymous reviewers for their valuable comments on the earlier version of this paper.

Tawfeeq, Y. J. (2020). Mathematical modeling and numerical simulation of porous media single-phase fluid flow problem: a scientific review. International Research Journal of Engineering, IT \& Scientific Research, 6(4), 15-28. https://doi.org/10.21744/irjeis.v6n4.955 


\section{References}

Aarnes, J. E., Gimse, T., \& Lie, K. A. (2007). An introduction to the numerics of flow in porous media using Matlab. In Geometric modelling, numerical simulation, and optimization (pp. 265-306). Springer, Berlin, Heidelberg. https://doi.org/10.1007/978-3-540-68783-2_9

Ahmed, Tarek, H. (2010). Reservoir Eng. Handbook. 4th edition. Gulf Professional Publishing is an imprint of Elsevier, 30 Corporate Drive, Suite 400, Burlington, MA 01803, USA. The Boulevard, Langford Lane, Kidlington, Oxford, OX5 1GB, U.K.

Aziz, K. (1979). Petroleum reservoir simulation. Applied Science Publishers, 476.

Bear, J. (1988). Dynamics of fluids in porous media Dover Publications. INC, New York.

Chen, Z., Huan, G., \& Ma, Y. (2006). Computational methods for multiphase flows in porous media. Society for Industrial and Applied Mathematics.

Ertekin T., Jamal H. Abou-Kassem \& Gregory R. King. (2001). Basic Applied Reservoir Simulation. Henry L. Doherty Memorial Fund of AIME. Society of Petroleum Engineers. Richardson, Texas

Ewing, R. E. (1983). Problems arising in the modeling of processes for hydrocarbon recovery. In The mathematics of reservoir simulation (pp. 3-34). Society for Industrial and Applied Mathematics. https://doi.org/10.1137/1.9781611971071.ch1

Fanchi, J. R. (2001). Principles of Applied Reservoir Simulation. 2nd Edition. Vol. I. United States of America: Gulf Professional Publishing.

Fanchi, J.R. (2006). Principles of applied reservoir simulation, 3rd ed., Gulf Professional. Publishing, Burlington.

Gerritsen, M. G., \& Durlofsky, L. J. (2005). Modeling fluid flow in oil reservoirs. Annu. Rev. Fluid Mech., 37, 211238. https://doi.org/10.1146/annurev.fluid.37.061903.175748

Baker, H. A., \& Awad, A. S. (2017). Reservoir Characterizations and Reservoir Performance of Mishrif Formation in Amara Oil Field. Journal of Engineering, 23(12), 33-50.

Jansen, J. D. (2013). A systems description of flow through porous media (Vol. 570). New York: Springer.

Cosentino, L. (2001). Integrated reservoir studies. Editions Technip.

Mattax, C. C., \& Dalton, R. L. (1990). Reservoir Simulation (includes associated papers 21606 and 21620). Journal of Petroleum Technology, 42(06), 692-695. https://doi.org/10.2118/20399-PA

Mohammed, M. A., Hamd-Allah, S., \& Hameed, R. (2020). Diagnosing Water Problem for Asmari Reservoir in Abu Ghirab Oilfield Using Analytical and Numerical Approaches. Journal of Engineering, 26(4), 94-110. https://doi.org/10.31026/j.eng.2020.04.07

Odeh, A. S. (1982). An overview of mathematical modeling of the behavior of hydrocarbon reservoirs. Siam Review, 24(3), 263-273. https://doi.org/10.1137/1024062

Peaceman, D. W. (2000). Fundamentals of numerical reservoir simulation. Elsevier. 